\title{
Open Mathematics
}

\author{
Research Article
}

Joanna Jureczko*

\section{The new operations on complete ideals}

https://doi.org/10.1515/math-2019-0032

Received May 13, 2018; accepted February 21, 2019

Abstract: We introduce the notion of K-ideals associated with Kuratowski partitions. Using new operations on complete ideals we show connections between K-ideals and precipitous ideals and prove that every complete ideal can be represented by some K-ideal.

Keywords: direct sum of spaces, Fréchet ideal, precipitous ideal, measurable cardinal,Lévy forcing, meager set, Baire property, K-ideal, Kuratowski partition.

MSC: Primary 03C25, 03E35, 03E55, 03C20, 54E52

\section{Introduction}

The main idea of this paper is to show some operations on complete ideals associated with so called Kuratowski partitions. As will be shown, it is not enough to use some operations on these ideals only. It requires using some properties of topology of given spaces for which the Kuratowski partitions and hence these ideals exist.

The concept of Kuratowski partitions emerged when attempting to solve the problem set by K. Kuratowski, in [1], whether each function $f: X \rightarrow Y$, from a completely metrizable space $X$ to a metrizable space $Y$, such that for each open set $V \subset Y$ the set $f^{-1}(V)$ has the Baire property (i.e. it differs from an open set by a meager set) is continuous apart from a meager set.

As shown by A. Emeryk, R. Frankiewicz and W. Kulpa, in [2, 3], this problem is equivalent to the problem of the non-existence of partitions of a metrizable space into meager sets with the property that the union of each its subfamily has the Baire property. A partition of a topological space with the above property is called Kuratowski partition. For the first time the above definition appeared in [4]. However, such partitions were considered frequently since the paper [2].

With any Kuratowski partition of a topological space into meager sets we associate an ideal called in this paper a $K$-ideal (see Section 2 for the formal definition). It seemed that the knowledge of such $K$-ideal could help to determine whether a given space admits Kuratowski partition. Unfortunately, it is not so, because as we will show, the structure of such $K$-ideal can be a Fréchet ideal so not precipitous or includes a Fréchet ideal and be a subideal of some complete ideal (not necessary proper). For "decoding" Kuratowski partition from a given $K$-ideal we need more information about topology of the space in which we consider such an ideal. So, as we will show in Theorem 1 and Theorem 2, no characterisation of a space given by a $K$-ideal is possible.

The motivation for our considerations has been the result published in 1987 by R. Frankiewicz and K. Kunen in [5], who showed that the existence of Kuratowski partitions is equiconsistent with the existence of measurable cardinals. So, the assumption of the existence of Kuratowski partition is as strong as the existence of a measurable cardinal. It is worth to add that the proof of this fact, presented in [5], relied on the forcing

${ }^{\star}$ Corresponding Author: Joanna Jureczko: Wrocław University of Science and Technology, Wrocław, Poland,

E-mail: joanna.jureczko@pwr.edu.pl 
method and till now only such a proof has been known (but in [6] this result is obtain without using any metamathematical methods).

Our second motivation for considering the operations on $K$-ideals has been the example of non-separable Baire spaces for which the product is non-Baire, (see [7]).

In this paper we also come back to the following problem: assume that $X$ is a topological space and $Y$ is its subspace and assume that $Y$ admits Kuratowski partition. Does $\mathrm{X}$ also admit Kuratowski partition? This problem was examined by several researchers. Some particular results were obtained in $[8,9]$ but only for Borel sets. In Theorem 3 we give a negative answer. More precisely, we show that the completion of some metric Baire space with Kuratowski partition does not have Kuratowski partition. And again, the motivation for consideration an incomplete metric Baire space with Kuratowski partition comes from [5], where the authors consider the existence of such partitions for complete and incomplete metric Baire spaces.

\section{Definitions and basic facts}

In this part we give basic definitions and previous results used in the next section of this paper. Other notations of this paper are recognized as standard for the area and can be found in [10] (infinite combinatorics, forcing), [11, 12] (topology).

I. Let $X$ be a topological space. A subset $A \subseteq X$ is said to have the Baire property if there exists a meager set $M \subset X$ and an open set $U \subset X$ such that $A=U \triangle M$, where $\triangle$ means a symmetric difference of sets.

Let $X$ be a topological space, $\mathcal{F}$ be a partition of $X$ into meager sets. We say that $\mathcal{F}$ is Kuratowski partition on $X$ if $\cup \mathcal{F}^{\prime}$ has the Baire property for each $\mathcal{F}^{\prime} \subseteq \mathcal{F}$.

Enumerate

$$
\mathcal{F}=\left\langle F_{\alpha}: \alpha<\kappa\right\rangle \text {. }
$$

(We will consider Kuratowski partitions as sequences of sets, because in further parts of this paper we will operate on their enumerations).

With any Kuratowski partition $\mathcal{F}$ we associate the ideal

$$
I_{\mathcal{F}}=\left\{A \subset \kappa: \bigcup_{\alpha \in A} F_{\alpha} \text { is meager }\right\}
$$

which we call $K$-ideal associated with $\mathcal{F}$.

As defined above, Kuratowski partition $\mathcal{F}$ of a topological space $X$ is indexed by ordinals, but the $K$-ideal associated with $\mathcal{F}$ is a $\kappa$-complete ideal on cardinals. Thus in some results of this paper the considerations are carried out on cardinals instead of topological spaces.

II. Let $\kappa$ be an uncountable regular cardinal and let $I$ be an ideal on $\kappa$, (a family of sets of $P(\kappa)$ closed under taking finite unions and subsets.). Let $S$ be a set of positive measure, i.e. $S \in \mathcal{P}(\kappa) \backslash I=I^{+}$. An $I$-partition of $S$ is a maximal family $W$ of subsets of $S$ of positive measure such that $A \cap B \in I$ for all distinct $A, B \in W$. An $I$-partition $W_{1}$ of $S$ is a refinement of an $I$-partition $W_{2}$ of $S$, if each $A \in W_{1}$ is a subset of some $B \in W_{2}$. In this case we will write $W_{1} \leq W_{2}$.

Let $I$ be a $\kappa$-complete ideal on $\kappa$ containing singletons. The ideal $I$ is precipitous if whenever $S \in I^{+}$and $\left\{W_{n}: n<\omega\right\}$ are $I$ - partitions of $S$ such that $W_{0} \geq W_{1} \geq \ldots \geq W_{n} \geq \ldots$, then there exists a sequence of sets $A_{0} \supseteq A_{1} \supseteq \ldots \supseteq A_{n} \supseteq \ldots$ such that $A_{n} \in W_{n}$ for each $n$, and $\bigcap_{n=0}^{\infty} A_{n}$ is nonempty. (see also [10, p. 438-439]).

III. Let $\lambda \geq \omega$ be a cardinal. Then

$$
[\lambda]^{<\lambda}=\{A \subset \lambda:|A|<\lambda\}
$$

is the Fréchet ideal on $\lambda$. 
Fact 1. ([10, Lemma 22.20, p. 425]) Let $\kappa$ be an uncountable regular cardinal. Then $[\kappa]^{<\kappa}$ is not precipitous.

IV. An uncountable regular cardinal $\kappa$ is called measurable if there exists a nontrivial maximal $\kappa$-complete ideal on $\kappa$, or equivalently, if there exists a nonprincipal $\kappa$-complete ultrafilter on $\kappa$.

\section{Fact 2. ([10, Theorem 22.33, p. 432])}

(a) If $\kappa$ is a regular uncountable cardinal that carries a precipitous ideal, then $\kappa$ is measurable in an inner model of $Z F C$

(b) If $\kappa$ is measurable cardinal, then there exists a generic extension in which $\kappa=\aleph_{1}$, and $\kappa$ carries a precipitous ideal.

Fact 3. ([5, Theorems 3.3 and 3.4]) ZFC + "there is measurable cardinal" is equiconsistent with ZFC + "there is Kuratowski partition of Baire space $X^{\prime \prime}$.

A cardinal $\kappa$ is called inaccessible iff $\kappa$ is regular uncountable and

$$
2^{\lambda}<\kappa \text { for all } \lambda<\kappa .
$$

Fact 4. ([10, Lemma 10.4, p. 127]) Every measurable cardinal is inaccessible.

V. If $\lambda$ is a cardinal, then let $(D(\lambda))^{\omega}$ denotes a metric space, where $D(\lambda)$ is a discrete space of cardinality $\lambda$. For each $n \in \omega$ and function $x: n \rightarrow \kappa$ let $U(x)=\left\{f \in^{\omega} \lambda: f \mid n=x\right\}$. The family $\{U(x): x: n \rightarrow \kappa, n \in \omega\}$ is a canonical base for $(D(\lambda))^{\omega}$, (see [5]).

Fact 5. ([5, Theorem 2.1]) Let $J$ be an $\omega_{1}$-complete ultrafilter on $\kappa$. Then $\left(D\left(2^{\kappa}\right)\right)^{\omega}$ can be split into $\kappa$ meager sets $\left\{F_{\alpha}: \alpha<\kappa\right\}$ in such a way that for each $A \subseteq \kappa$ the set $\bigcup_{\alpha \in A} F_{\alpha}$ has the Baire property.

Remark 1. The elements of the partition in Fact 5 can be of the form

$$
F_{\alpha}=\left\{x \in\left(D\left(2^{\omega}\right)\right)^{\omega}: \alpha=\min \bigcap\{x(n): n \in \omega\}\right\},
$$

(see the proof of [5, Theorem 2.1]).

VI. Let $I$ be an ideal on $\kappa$. Let

$$
X(I)=\left\{x \in\left(I^{+}\right)^{\omega}: \bigcap\{x(n): n \in \omega\} \neq \emptyset \text { and } \forall n \in \omega \bigcap\{x(m): m<n\} \in I^{+}\right\} .
$$

As was pointed out in [5], the set $X(I)$ is considered as a subset of a complete metric space $\left(I^{+}\right)^{\omega}$, where $I^{+}$is equipped with the discrete topology.

Fact 6. ([5, Proposition 3.1]) $X(I)$ is a Baire space iff I is a precipitous ideal.

Fact 7. ([5, Theorem 3.2]) Let I be a precipitous ideal on some regular cardinal. Then there is a Kuratowski partition of the metric Baire space $X(I)$.

VII. Let $\left\{X_{S}\right\}_{s \in S}$ be the family of pairwise disjoint topological spaces. Consider the set $X=\bigcup_{s \in S} X_{S}$ and the family $\mathcal{O}$ of all open sets $U \subseteq X$ such that $U \cap X_{S}$ is open in $X_{S}$ for every $s \in S$. Obviously $\mathcal{O}$ is a topology on $X$. The set $X$ with this topology is called direct sum of $\left\{X_{s}\right\}_{s \in S}$ and is denoted by $\oplus_{s \in S} X_{s}$. A set $A \subseteq \oplus_{s \in S} X_{S}$ is closed iff $A \cap X_{S}$ is closed in $X_{S}$ for every $s \in S$. Thus, all sets $X_{s}$ are open-and-closed in $\oplus_{s \in S} X_{S}$, (see [11, p. 74-75]).

In the presence of Fact 8, (see below), we can define meager sets in $\oplus_{s \in S} X_{S}$ similarly to defining open and closed sets. 
Fact 8. ([12, Union Theorem, p. 82][13]) If $\left\{X_{s}\right\}_{s \in S}$ is a family (of an arbitrary power) of sets open relative to the union $\bigcup_{s \in S} X_{S}$ and if each $X_{S}$ is meager, then $\bigcup_{s \in S} X_{S}$ is also meager.

Notice that $\oplus_{s \in S} X_{S}$ can also be defined for a family of topological spaces $\left\{X_{S}\right\}_{s \in S}$ which are not pairwise disjoint. In this case one should take a family $\left\{X_{s}^{\prime}\right\}_{s \in S}$ of pairwise disjoint spaces such that $X_{S}^{\prime}$ is homeomorphic to $X_{s}$ for any $s \in S$, (e.g. one can take $X_{s}^{\prime}=X_{S} \times\{s\}$ with the topology generated by the mapping $p_{s}: X_{S}^{\prime} \rightarrow X_{s}$, where $\left.p_{s}(x, s)=x\right)$, and define $\oplus_{s \in S} X_{S}=\oplus_{s \in S} X_{s}^{\prime}$,

VIII. The Lévy Collapse.

Fact 9. ([10, Theorem 15.22 (Lévy's Theorem), p. 238]) Let $\lambda$ be a regular cardinal and let $\kappa>\lambda$ be an inaccessible cardinal. There is a notion of forcing $(P,<)$ such that:

(i) every $\alpha$ such that $\lambda \leqslant \alpha<\kappa$ has cardinality $\lambda$ in $V[G]$, and

(ii) every cardinal $\leqslant \lambda$ and every cardinal $\geqslant \kappa$ remains a cardinal in $V[G]$. Hence $V[G] \models \kappa=\lambda^{+}$.

IX. Let $\kappa$ be a measurable cardinal and let $U$ be a nonprincipal $\kappa$-complete ultrafilter on $\kappa$. Let us consider the model $L[U]$ of all sets constructible from $U$.

Fact 10. ([10, Theorem 19.3 (Silver's Theorem), p. 339 and Lemma 19.20, p. 351]) If $V=L[U]$, then $G C H$ holds.

Fact 11. ([10, Theorem 19.14 (Kunen's Theorem), p. 348 and Lemma 19.20, p. 351]) In $L[U] \kappa$ is the only measurable. $L[U]$ is the smallest model in which $\kappa$ is measurable.

X. Let $X$ be a space. Then the weight of $X$ is defined as follows

$$
w(X)=\min \{|\mathcal{B}|: \mathcal{B} \text { is a base for } X\}+\omega .
$$

A $\pi$-base for $X$ is a collection $\mathcal{V}$ of non-empty open sets in $X$ such that if $U \subseteq X$ is a non-empty open set, then $V \subseteq U$ for some $V \in \mathcal{V}$.

The $\pi$-weight of $X$ is defined as follows

$$
\pi w(X)=\min \{|\mathcal{V}|: \mathcal{V} \text { is a } \pi \text {-base for } X\}+\omega .
$$

Fact 12. ([14, Theorem 8.1, p. 32-33]) For an infinite metrizable space

(a) $w(X)=\pi w(X)$,

(b) $w(X) \leqslant|X| \leqslant(w(X))^{\omega}$.

XI. A space $X$ is a Čech complete space if $X$ is a dense $G_{\delta}$ subset of a compact space, (see [11, p. 196]). Each Čech complete space is a Baire space.

Fact 13. ([3]) Let $X$ be a Čech complete space such that $\pi w(X) \leq 2^{\omega}$. Then there are no Kuratowski partitions on $X$.

XII. For a given metric space $X$, by $\tilde{X}$ we denote its completion in the sense of Fact 14, (see below).

Fact 14. ([11, Theorem 4.3.19, p. 272]) For every metric space $(X, \rho)$ there exists exactly one (up to isometry) complete metric space $(\tilde{X}, \tilde{\rho})$ such that $\tilde{X}$ contains a dense subspace isometric to $(X, \rho)$. Moreover we have

$$
w(\tilde{X})=w(X) .
$$




\section{Main results}

The first two theorems of this section concern some properties of $K$-ideals. As will be shown below, such a $K$-ideal can be Fréchet ideal and every $\kappa$-complete ideal can be "represented" by some $K$-ideal. In the proofs of both theorems we use new construction of ideals relying on enlarging of the space admitting Kuratowski partition as direct sum of some its copies, (i.e. its homeomorphic spaces).

Theorem 1. Let $Y$ be a Baire space. Let $X \subset Y$ be a Baire space with Kuratowski partition $\mathcal{F}$ such that

(i) $|\mathcal{F}|=\kappa$, where $\kappa=\min \{|\mathcal{F}|: \mathcal{F}$ is Kuratowski partition of $X\}$ is regular uncountable cardinal,

(ii) $\bigcup \mathcal{F}^{\prime}$ is meager for any $\mathcal{F}^{\prime} \subset \mathcal{F}$ of cardinality $<\kappa$.

Let $\Pi$ be a family of all permutations of $\kappa$. Then the direct sum $\oplus_{\pi \in \Pi} X_{\pi}$ has Kuratowski partition $\mathcal{F}^{*}$ and $K$-ideal $I_{\mathcal{F}^{\star}}$ associated with $\mathcal{F}^{\star}$ is $[\kappa]^{<\kappa}$.

Proof. Let $\mathcal{F}=\left\langle F_{\alpha}: \alpha<\kappa\right\rangle$ be Kuratowski partition of $X$. Consider the set $\Pi$ of all permutations of $\kappa$, (i.e. $\Pi=\kappa !)$. Let $\left\{X_{\pi}: \pi \in \Pi\right\}$ be a set of spaces homeomorphic to $X$ indexed by elements of $\Pi$. Consider the direct sum $\oplus_{\pi \in \Pi} X_{\pi}$. Of course each $X_{\pi}$ is open in $\oplus_{\pi \in \Pi} X_{\pi}$. For each $\pi \in \Pi$ let $\mathcal{F}_{\pi}$ be the partition of $X_{\pi}$ such that

$$
\mathcal{F}_{\pi}=\left\langle F_{\pi(\alpha)}: \alpha<\kappa\right\rangle .
$$

(In fact we use above a copy of $F_{\pi(\alpha)}$ inside of $X_{\pi}$ ).

Such a family is Kuratowski partition of $X_{\pi}$. For each $\alpha<\kappa$ consider

$$
F^{\star}(\alpha)=\bigcup\left\{F_{\pi(\alpha)}: \pi \in \Pi\right\} .
$$

Then by Fact 8 the set $F^{\star}(\alpha)$ is meager in $\oplus_{\pi \in \Pi} X_{\pi}$ and

$$
\mathcal{F}^{\star}=\left\langle F^{\star}(\alpha): \alpha<\kappa\right\rangle
$$

is Kuratowski partition of $\oplus_{\pi \in \Pi} X_{\pi}$.

Let $I_{\mathcal{F}^{\star}}$ be $K$-ideal associated with $\mathcal{F}^{\star}$. By $(i i)[\kappa]^{<\kappa} \subset I_{\mathcal{F}^{*}}$. Observe that there are $A \subset \kappa,|A|=\kappa$ such that $A \notin I_{\mathcal{F}^{\star}}$. If not, then $\bigcup_{\alpha \in A} F^{\star}(\alpha)$ is meager for any $A \subset \kappa$ of cardinality $\kappa$. By Fact $8, \bigcup_{A \subset \kappa} \bigcup_{\alpha \in A} F^{\star}(\alpha)$ would be meager. But it is impossible, because $X$ is a Baire space. Now we show that no $A \subset \kappa$ of cardinality $\kappa$ belongs to $I_{\mathcal{F}^{*}}$. Suppose that there exists $A_{0} \in I_{\mathcal{F}^{*}}$ such that $\left|A_{0}\right|=\kappa$. Then, there exists $B_{0} \subset \kappa$ of cardinality $\kappa$ such that $\left|A_{0} \cap B_{0}\right|=\kappa$ and $B_{0} \notin I_{\mathcal{F}^{*}}$. Take $\pi_{0} \in \Pi$ such that $\pi_{0}\left(A_{0}\right)=B_{0}$. Then $\bigcup_{\beta \in B_{0}} F_{\beta}^{\star}$ is non-meager. But by the construction above $\bigcup_{\beta \in B_{0}} F_{\beta}^{\star}=\bigcup_{\beta \in \pi_{0}\left(A_{0}\right)} F_{\beta}^{\star}=\bigcup_{\alpha \in A_{0}} F_{\pi_{0}(\alpha)}^{\star}=\bigcup_{\alpha \in A_{0}} F_{\alpha}^{\star}$. A contradiction.

By Fact 3, one can suppose that large cardinals and $K$-ideals are strongly related, but comparing Theorem 1 and Fact 1 one can conclude that such a $K$-ideal does not have to be necessary precipitous (see also Fact 2 ).

In the next theorem we construct the space for which a given complete ideal is a $K$-ideal. We will also use a similar construction as in the proof of Theorem 1, i.e. enlarging the space by adding some of its copies only. The difference is the proof of Theorem 1 we enlarge space in order to make the desired union non-meager. But in the proof of Theorem 2 we enlarge space in order to "eliminate" non-meager unions by "replacement"it by meager unions.

Notice that in the next theorem the assumption that $\kappa$ is measurable is essential (compare Fact 3). What is more, we assume that $\kappa$ is the least measurable cardinal, because there can be many measurable cardinals according to the Ulam Theorem ([10, Theorem 10.1, p. 126 ], more precisely [10, Lemma 10.5, p 128]).

Theorem 2. Assume that ZFC+"there exists a measurable cardinal" is consistent. Let $\kappa$ be the least measurable cardinal. Then for each $\kappa$-complete ideal I on $\kappa$ such that $[\kappa]^{<\kappa} \subseteq$ I there exists a space with a Kuratowski partition $\mathcal{F}^{\#}$ of cardinality $\kappa$ such that $I$ is of the form $I_{\mathcal{F}^{\#}}$.

Proof. By assumption, there exists a maximal non-trivial and $\kappa$-complete ideal $I$ on $\kappa$ :

$$
I=\left\{B_{\xi} \subseteq \kappa: \xi<2^{\kappa}\right\} .
$$


Let $J$ be a dual filter to $I$, i.e.

$$
J=\left\{P_{\xi}: P_{\xi}=\kappa \backslash B_{\xi} \text { for some } B_{\xi} \in I, \xi<2^{\kappa}\right\}
$$

Obviously, $J$ is a nonprincipal $\kappa$-complete ultrafilter.

Consider a metric space $(J)^{\omega}$, where $J$ is equipped with a discrete topology. Since $(J)^{\omega}$ is complete metric hence is a Baire space.

For any $\alpha<\kappa$ define

$$
F_{\alpha}=\left\{x \in(J)^{\omega}: \alpha=\min \bigcap\{x(n): n \in \omega\}\right\}
$$

and take $\mathcal{F}=\left\langle F_{\alpha}: \alpha<\kappa\right\rangle$. By Fact 5, $\mathcal{F}$ is a Kuratowski partition of $(J)^{\omega}$. Let $I_{\mathcal{F}}$ be a $K$-ideal associated with $\mathcal{F}$.

Now turn to the ideal $I$. For each $B_{\xi} \in I$ we associate the following union $\bigcup_{\alpha \in B_{\xi}} F_{\alpha}$, where $F_{\alpha} \in \mathcal{F}$. Obviously such a union can be meager or non-meager. To complete the proof we enlarge the space $(J)^{\omega}$ by adding some of its copies, to "eliminate" the case when the above union, (i.e. $\bigcup_{\alpha \in B_{\xi}} F_{\alpha}$ ) is non-meager. In fact we "replace" such union by another which is meager. We obtain this by the following way.

Since $[\kappa]^{<\kappa} \subseteq I$ and in the presence of Theorem 1 the union $\bigcup_{\alpha \in B} F_{\alpha}$ is meager for each $B \in[\kappa]^{<\kappa}$.

Consider

$$
\begin{gathered}
I^{\prime}=\left\{B \in I:|B|=\kappa, \bigcup_{\beta \in B} F_{\beta} \text { is meager }\right\} . \\
I^{\prime \prime}=\left\{A \in I:|A|=\kappa, \bigcup_{\alpha \in A} F_{\alpha} \text { is non-meager }\right\} .
\end{gathered}
$$

Obviously both families are non-empty, because $(J)^{\omega}$ is a Baire space.

Let $B \in I^{\prime}$. Then $\bigcup_{\alpha \in B} F_{\alpha}=(J)^{\omega} \backslash \bigcup_{\alpha \in \kappa \backslash B} F_{\alpha}$. By Fact 5, $\bigcup_{\alpha \in \kappa \backslash B} F_{\alpha}$ contains an open set, therefore it is comeager, so $\bigcup_{\alpha \in B} F_{\alpha}$ is meager. This means that $I \subseteq I_{\mathcal{F}}$ and since $I$ is maximal, $I=I_{\mathcal{F}}$.

For any $A \in I^{\prime \prime}$ take $B \in I^{\prime}$ and a permutation $\pi_{B}^{A} \in \kappa$ ! such that $\pi_{B}^{A}(A)=B$. Define

$$
\Pi(I)=\left\{\pi \in \kappa !: \pi=\pi_{B}^{A}, B \in I^{\prime}, A \in I^{\prime \prime}\right\} .
$$

Now for each $\pi \in \Pi(I)$ take

$$
\mathcal{F}_{\pi}=\left\langle F_{\pi(\alpha)}: \alpha<\kappa\right\rangle .
$$

Such a family is Kuratowski partition of $\left((J)^{\omega}\right)_{\pi}$. Now for each $\alpha<\kappa$ take

$$
F^{\#}(\alpha)=\bigcup\left\{F_{\pi(\alpha)}: \pi \in \Pi(I)\right\}
$$

By Fact 8 , each $F^{\#}(\alpha)$ is meager in $\oplus_{\pi \in \Pi(I)}\left((J)^{\omega}\right)_{\pi}$. Then the family

$$
\mathcal{F}^{\#}=\left\langle F^{\#}(\alpha): \pi \in \Pi(I)\right\rangle
$$

is Kuratowski partition in $\oplus_{\pi \in \Pi(I)}\left((J)^{\omega}\right)_{\pi}$. Let $I_{\mathcal{F}^{\#}}$ be a $K$-ideal associated with $\mathcal{F}^{\#}$. Then $I_{\mathcal{F}^{\#}} \subseteq I$. By previous considerations we have

$$
I_{\mathcal{F} \#} \cup I^{\prime} \cup[\kappa]^{<\kappa}=I .
$$

Note, that if $\kappa$ is nonmeasurable but there exists Kuratowski partition of cardinality $\kappa$ of a space $X$ then one can obtain both the Fréchet ideal (as was shown in Theorem 1) or the $\kappa$-complete ideal containing the Fréchet ideal and included in the $K$-ideal of some space as was shown in Theorem 2. So, as was announced in Introduction, no characterisation of spaces with Kuratowski partitions by $K$-ideals is possible.

In the next theorem, we show that "enlarging" the Kuratowski partition of some space to its "superspace" while preserving its size is not possible. More precisely, we show that under assumptions "there exists a precipitous ideal on $\omega_{1}$ " and Lusin Hypothesis: " $2^{\omega_{0}}=2^{\omega_{1}}$ ", there exists an incomplete metric Baire space with Kuratowski partition for which the completion does not admit such a partition. Notice that these two assumptions are consistent if $Z F C+$ "there exists measurable cardinal" is consistent. More precisely we have the following proposition. 
Proposition 1. Assume that $Z F C+$ "there exists measurable cardinal" is consistent. Then $Z F C+{ }^{\prime \omega}=2^{\omega_{1}}$ " + "there exists a precipitous ideal on $\omega_{1}$ " is consistent.

Proof. Assume that ZFC + "there exists a measurable cardinal" is consistent. Consider the model $V=L[U]$, (see Section 2.X). By Fact 10, GCH holds in $V$, by Fact 11, there is only one measurable cardinal in $V$. Name it by $\kappa$. By Fact 4, $\kappa$ is inaccessible. Now use the Lévy Collapse, i.e. Fact 9 applied to $\lambda=\omega_{1}$ obtaining $\kappa=\omega_{1}$ and $\kappa^{+}=\omega_{2}$ in $V\left[G_{1}\right]$.

Now, add $\omega_{2}$ Cohen reals with finite support, (see e.g. [10, p. 225-226]) obtaining the model $V\left[G_{1}\right]\left[G_{2}\right]$ which preserves all cardinals (because Cohen forcing is c.c.c). Hence $V\left[G_{1}\right]\left[G_{2}\right] \models 2^{\omega_{0}}=\omega_{2}$, (see [10, p. 226]) and $V\left[G_{1}\right]\left[G_{2}\right]=2^{\omega_{1}}=\omega_{2}$ (because in this model GCH holds above $\omega_{1}$ ). Hence

$$
V\left[G_{1}\right]\left[G_{2}\right]=2^{\omega_{0}}=2^{\omega_{1}} .
$$

Now, by [15, Remark, p. 604] we obtain that adding $\omega_{2}$ Cohen reals does not have influence on measurability, i.e. By Fact 2(b), in $V\left[G_{1}\right]\left[G_{2}\right]$ there is a precipitous ideal on $\omega_{1}$.

Note that using Cohen forcing, (i.e. adding $\omega_{2}$ Cohen reals in our case) is the simpliest way to prove Proposition 1.

Now we are ready to prove the announced theorem.

Theorem 3. Assume that ZFC + "there exists a measurable cardinal" is consistent. Then there exists a metric Baire space with Kuratowski partition for which a completion does not have a Kuratowski partition.

Proof. By Proposition 1, there exists a model in ZFC in which ZFC+ "2 ${ }^{\omega_{0}}=2^{\omega_{1}} "+$ "there exists a precipitous ideal on $\omega_{1}$ " is consistent. Let $I$ be a precipitous ideal on $\omega_{1}$. Let $X(I)$ be as defined in Section 2.VI. By Fact 6, $X(I)$ is a Baire space and by Fact 7, $X(I)$ has a Kuratowski partition. By Fact $12(\mathrm{~b}) w(X(I))=2^{\omega_{1}}$. Consider $\tilde{X}(I)$ - the completion of $X(I)$ (in the sense of Fact 14). By Fact 14, we have that $w(X(I))=w(\tilde{X}(I))$. Hence $w(\tilde{X}(I))=2^{\omega_{1}}$. By Fact 12(a) we have that $w(\tilde{X}(I))=\pi w(\tilde{X}(I))$. Hence $\pi w(\tilde{X}(I))=2^{\omega_{1}}$. But by our assumptions that $2^{\omega_{0}}=2^{\omega_{1}}$ we have that $\pi w(\tilde{X}(I))=2^{\omega_{0}}$. By Fact 13, $\tilde{X}(I)$ does not admit Kuratowski partition.

Note, that in Theorem 3 we do not need to assume that $X$ is a metric nonseparable Baire space because, as was shown in [4, Lemma 5 and Lemma 6], if a Kuratowski partition exists for a Hausdorff Baire space, then it also exists for a metric space.

The model $L[U]$, (rather its generic extension, see also [5]), can also be used in the considerations presented in this paper, because these methods can give us other, more "complicated" structures. In such obtained structures one can also investigate the existence of Kuratowski partitions. These and similar considerations will be the part of our next paper in this area.

Acknowledgement: The author is very grateful to the Reviewers for their comments which have raised the quality of this text and allowed to avoid many errors and inaccuracies.

\section{References}

[1] Kuratowski K., Quelques problemés concernant les espaces métriques nonseparables, Fund. Math., 1935, 25, 534-545

[2] Emeryk A., Frankiewicz R., Kulpa W., On functions having the Baire property, Bull. Ac. Pol.: Math., 1979, 27, 489-491

[3] Emeryk A., Frankiewicz R., Kulpa W., Remarks on Kuratowski’s Theorem on Meager Sets, Bull. Ac. Pol.: Math., 1979, 27, 493-498

[4] Frankiewicz R., Szczepaniak S., On partitions of Ellentuck-large sets, Top. App., 2014, 167, 80 âĂŞ-86

[5] Frankiewicz R., Kunen K., Solutions of Kuratowski's problem on functions having the Baire property, I, Fund. Math., 1987, 128, 171-180

[6] Frankiewicz R., Jureczko J., The existence of Kuratowski partitions directly equivalent to the existence of large cardinals, (to appear)

[7] Fleissner W. G., Kunen K., Barely Baire spaces, Fund. Math., 1987, 101(3), 229-240 
[8] Fremlin D. H., Hansell R. W. and J. Junilla H., Borel functions of bounded class, Trans AMS, 1983, 277(2), 835-849

[9] Kaniewski J., Pol R., Borel-measurable Selectors for Compact-valued mappings in the Non-separable Case, Bull. Ac. Pol.: Math., 1975, 23(10), 1043-1050

[10] Jech T., Set Theory: The Third Millennium Edition, revised and expanded, 2003, Springer

[11] Engelking R., General Topology (Revised and completed edition), 1989, Heldermann Verlag Berlin

[12] Kuratowski K., Topology, vol. 1, 1966, Academic Press, New York and London

[13] Banach S., Théoréme sur les ensembles de premiére catégorie, Fund. Math., 1930, 16, 395-398

[14] Hodel R., K. Kunen et al. (Eds.), Handbook of Set-Theoretic Topology, 1984, R. Hodel,Cardinal Functions I, 2-61, Amsterdam, Elsevier Science Publishers

[15] Baumgartner J. E., Taylor A. D., Saturation properties of ideals in generic extensions. II, Trans. AMS, 1982, 271(2), 587-609 